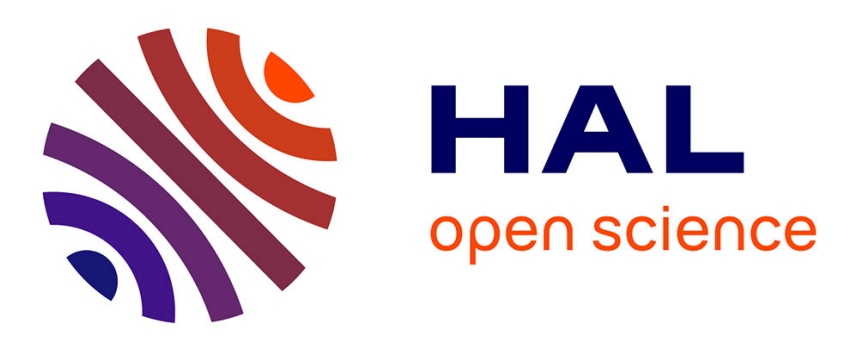

\title{
Solution of large deformation impact problems with friction between Blatz-Ko hyperelastic bodies
}

Zhi-Qiang Feng, Benoit Magnain, Jean-Michel Cros

\section{To cite this version:}

Zhi-Qiang Feng, Benoit Magnain, Jean-Michel Cros. Solution of large deformation impact problems with friction between Blatz-Ko hyperelastic bodies. International Journal of Engineering Science, 2006, 44 (1-2), pp.113-126. 10.1016/j.ijengsci.2005.06.006 . hal-01179157

\section{HAL Id: hal-01179157 \\ https://hal.science/hal-01179157}

Submitted on 28 Jun 2018

HAL is a multi-disciplinary open access archive for the deposit and dissemination of scientific research documents, whether they are published or not. The documents may come from teaching and research institutions in France or abroad, or from public or private research centers.
L'archive ouverte pluridisciplinaire HAL, est destinée au dépôt et à la diffusion de documents scientifiques de niveau recherche, publiés ou non, émanant des établissements d'enseignement et de recherche français ou étrangers, des laboratoires publics ou privés. 


\title{
Solution of large deformation impact problems with friction between Blatz-Ko hyperelastic bodies
}

\author{
Zhi-Qiang Feng*, Benoît Magnain, Jean-Michel Cros \\ Laboratoire de Mécanique et d'Energétique d'Evry, Université d’Evry-Val d'Essonne, 40 rue du Pelvoux, F91020 Evry Cedex, France
}

Feng et al. [Z.Q. Feng, F. Peyraut, N. Labed, Solution of large deformation contact problems with friction between Blatz-Ko hyperelastic bodies, Int. J. Eng. Sci. 41 (2003) 2213-2225] have proposed a study of contact problems between Blatz-Ko hyperelastic bodies in static cases using the bi-potential method. The extension of this method for dynamic analysis of impact problems is realized in the present work. The total Lagrangian formulation is adopted to describe large strains and large displacements non-linear behavior. A first order algorithm is applied for the numerical integration of the time-discretized equation of motion. Numerical examples are carried out in two cases: rigid-deformable and deformable-deformable-rigid impacts in 2D. Numerical results show that the proposed approach is robust and efficient and the physical energy dissipation phenomena are apparently illustrated.

Keywords: Impact and friction; Hyperelastic large strains; Energy dissipation

\section{Introduction}

Problems involving contact and friction are among the most difficult ones in mechanics and at the same time of crucial practical importance in many engineering branches. A large number of algorithms for the modeling of contact problems by the finite element method have been presented in the literature. See for example the monographs by Kikuchi and Oden [2], Zhong [3] and Wriggers [4] and the references therein. De Saxcé and Feng [5] have proposed a bi-potential method, in which an augmented Lagrangian formulation was developed. Recently, Feng et al. [1] have successfully applied this method for the modeling of static contact problems between Blatz-Ko hyperelastic bodies.

For dynamic implicit analysis in structural mechanics, the most commonly used time integration algorithm is the second order algorithm such as Newmark, Wilson, HHT [6]. Hughes et al. [7] have presented a modified Newmark scheme for a class of contact-impact problems. The modification is based on some consideration of

\footnotetext{
* Corresponding author. Tel.: +3316947 75 01; fax: +3316947 7599.

E-mail address: feng@iup.univ-evry.fr (Z.-Q. Feng).
} 
wave propagation results. Wriggers et al. [8] have developed a radial return mapping scheme to deal with impact-contact problems. Armero and Petocz $[9,10]$ and Laursen et al. [11-13] have considered dynamic impact under the auspices of a conservative system and have proposed the means to address the dynamic contact conditions so that they preserve the global conservation properties. The integration scheme is based on the second order algorithm. Some first order algorithms have also been proposed by Zienkiewicz et al. [14] and Jean [15] for time stepping in structural dynamics.

The aim of the present paper is to apply the bi-potential method for contact modeling in dynamic cases involving large displacements and large hyperelastic strains using the first order algorithm for integration of the equation of motion. The algorithm developed is implemented into the finite element code FER/impact, using $\mathrm{C}++$ with object oriented programming techniques. Two numerical examples are performed in this study to show the validity of the model developed. The first example concerns the impact of a hyperelastic cylinder into a funnel. In order to show the physical energy dissipation by frictional effects, frictionless and frictional contact are considered for this example and the locking phenomenon is observed. The second example simulates the impact with jumping between two hyperelastic bodies and a rigid foundation.

\section{Problem setting}

\subsection{Contact kinematics}

In the following, basic definitions and notations used are described. Two deformable bodies $\mathscr{B}^{\alpha}$ (Fig. 1), $\alpha=1,2$, are considered. Each of them occupies the open, simply connected, bounded domain $\Omega^{\alpha} \subset \mathbb{R}^{3}$, whose generic point is denoted $\mathbf{X}^{\alpha}$. Furthermore, the solids are elastic and undergo large displacements. The boundary $\Gamma^{\alpha}$ of each body is assumed to be sufficiently smooth everywhere such that an outward unit normal vector, denoted by $\mathbf{n}^{\alpha}$, can be defined at any point $M$ on $\Gamma^{\alpha}$. At each time $t \in \mathbf{I}$, where $\mathbf{I}=[0, T]$ denotes the time interval corresponding to the loading process, the boundary $\Gamma^{\alpha}$ of the body $\mathscr{B}^{\alpha}$ can, in general, be split into three parts: $\Gamma_{\mathrm{u}}^{\alpha}$ with prescribed displacements $\overline{\mathbf{u}}^{\alpha}, \Gamma_{\mathrm{t}}^{\alpha}$ with prescribed boundary loads $\overline{\mathbf{t}}^{\alpha}$, and the potential contact surfaces $\Gamma_{\mathrm{c}}^{\alpha}$ where the two bodies $\mathscr{B}^{1}$ and $\mathscr{B}^{2}$ may possibly come into contact at some time $t$ (see Fig. 1):

$$
\Gamma^{\alpha}=\Gamma_{\mathrm{u}}^{\alpha} \cup \Gamma_{\mathrm{t}}^{\alpha} \cup \Gamma_{\mathrm{c}}^{\alpha}
$$

The successive deformed configurations of $\mathscr{B}^{\alpha}$ are described at each time $t$ by the displacement fields $\mathbf{u}^{\alpha}$ defined on $\bar{\Omega}^{\alpha}$ (i.e. the closure of $\left.\Omega^{\alpha}\right)$. On the contact surface, a unique normal $\mathbf{n}$ directed towards $\mathscr{B}^{1}\left(\mathbf{n} \equiv \mathbf{n}^{2}\right)$ is defined and the tangential plane, orthogonal to $\mathbf{n}$ in $\mathbb{R}^{3}$, is denoted by $\mathbf{T}$. To construct an orthonormal local basis, two unit vectors $\mathbf{t}_{x}$ and $\mathbf{t}_{y}$ are defined within the plane $\mathbf{T}$. For describing the frictional contact interactions that may occur on $\Gamma_{\mathrm{c}}$, we introduce the relative velocity with respect to $\mathscr{B}^{2}$

$$
\dot{\mathbf{u}}=\dot{\mathbf{u}}^{1}-\dot{\mathbf{u}}^{2}
$$

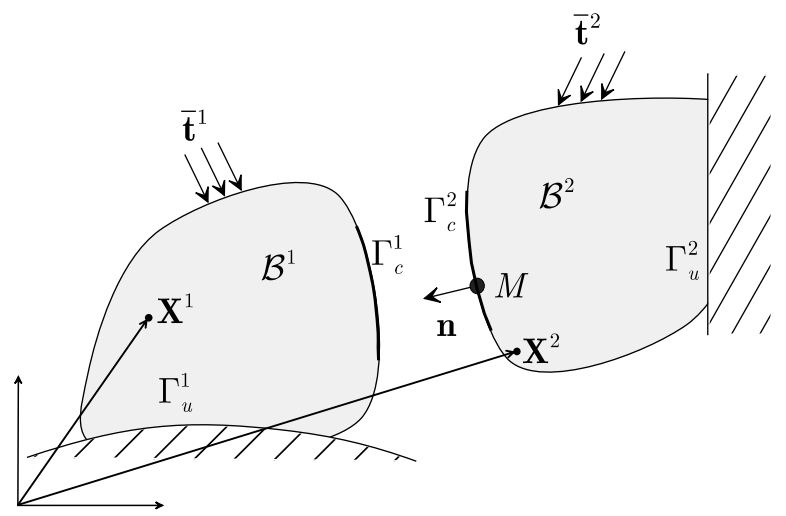

Fig. 1. Contact kinematics. 
where $\dot{\mathbf{u}}^{1}$ and $\dot{\mathbf{u}}^{2}$ are the instantaneous velocities of $\mathscr{B}^{1}$ and $\mathscr{B}^{2}$, respectively. Let $\mathbf{r}$ be the contact force distribution exerted on $\mathscr{B}^{1}$ at $M$ from $\mathscr{B}^{2}$. According to the action-reaction principle, $\mathscr{B}^{2}$ is subjected to the stress vector $-\mathbf{r}$. In the local coordinate system defined by the tangential plane $\mathbf{T}$ and the normal $\mathbf{n}$, any element $\dot{\mathbf{u}}$ and $\mathbf{r}$ may be uniquely decomposed as

$$
\begin{array}{ll}
\dot{\mathbf{u}}=\dot{\mathbf{u}}_{\mathrm{t}}+\dot{u}_{n} \mathbf{n}, \quad \dot{\mathbf{u}}_{\mathrm{t}} \in \mathbf{T}, \quad \dot{u}_{n} \in \mathbb{R} \\
\mathbf{r}=\mathbf{r}_{\mathrm{t}}+r_{n} \mathbf{n}, \quad \mathbf{r}_{\mathrm{t}} \in \mathbf{T}, \quad r_{n} \in \mathbb{R}
\end{array}
$$

\subsection{Contact law and friction rule}

The unilateral contact law is characterized by a geometric condition of non-penetration, a static condition of no-adhesion and a mechanical complementary condition. These three conditions are known as the Signorini conditions. The non-penetration condition constraints the displacement fields $\mathbf{u}^{\alpha}$ and is given by

$$
g(\mathbf{X})=\left(\mathbf{X}^{1}-\mathbf{X}^{2}\right) \cdot \mathbf{n} \geqslant 0
$$

where

$$
\mathbf{X}^{\alpha}(t)=\mathbf{X}^{\alpha}(t=0)+\mathbf{u}^{\alpha}
$$

The position vector $\mathbf{X}^{2}$ is found as the closest-point projection of the point $\mathbf{X}^{1} \in \Gamma_{\mathrm{c}}^{1}$ on the surface $\Gamma_{\mathrm{c}}^{2}$. Denoting by $h$ the initial gap obtained at the beginning of each time step.

$$
h=\left(\mathbf{X}^{1}-\mathbf{X}^{2}\right) \cdot \mathbf{n} \geqslant 0
$$

the impenetrability Signorini conditions are given by

$$
u_{n}+h \geqslant 0, \quad r_{n} \geqslant 0, \quad\left(u_{n}+h\right) r_{n}=0
$$

These conditions have to be satisfied at each time-instant $t \in \mathbf{I}$. Assume now that the bodies are initially in contact on a certain portion of $\Gamma_{\mathrm{c}}$. On this part of $\Gamma_{\mathrm{c}}$, the Signorini conditions turn into

$$
u_{n} \geqslant 0, \quad r_{n} \geqslant 0, \quad u_{n} r_{n}=0
$$

In general, at any time $t \in \mathbf{I}$, the potential contact surfaces $\Gamma_{\mathrm{c}}^{\alpha}$ can be split into two disjoint parts: ${ }^{+} \Gamma_{\mathrm{c}}$ where the bodies are already in contact and ${ }^{-} \Gamma_{\mathrm{c}}^{\alpha}$ where the bodies are not in contact:

$$
\Gamma_{\mathrm{c}}^{\alpha}={ }^{+} \Gamma_{\mathrm{c}} \cup{ }^{-} \Gamma_{\mathrm{c}}^{\alpha}
$$

In contrast to $\Gamma_{\mathrm{c}}^{\alpha},{ }^{+} \Gamma_{\mathrm{c}}$ and ${ }^{-} \Gamma_{\mathrm{c}}^{\alpha}$ change in time $t$ and can be empty at some $t \in \mathbf{I}$. We must stress that with the formulation (9) only a loss of contact is allowed and the extension of the contact area cannot be modelled with these relations. In the case of dynamic analysis such as impact problems, the Signorini conditions can be formulated, on ${ }^{+} \Gamma_{\mathrm{c}}$, in terms of relative velocity

$$
\dot{u}_{n} \geqslant 0, \quad r_{n} \geqslant 0, \quad \dot{u}_{n} r_{n}=0 \quad \text { on }^{+} \Gamma_{\mathrm{c}}
$$

when $\dot{u}_{n}>0$, the bodies are separating while they remain in contact for $\dot{u}_{n}=0$. The previous formulation of the Signorini conditions (11) can be combined with the sliding rule to derive the complete frictional contact law applicable on the contacting part of $\Gamma_{\mathrm{c}}$. This complete law specifies possible velocities of bodies that satisfy impenetrability, non-adhesion and the sliding rule. Obviously, for a strictly positive gap $\left(u_{n}>0\right)$, the normal relative velocity is arbitrary $\left(\dot{u}_{n} \in \mathbb{R}\right)$ and the normal reaction force is equal to zero $\left(r_{n}=0\right)$. Motions of bodies that are not in contact are arbitrary until contact is made. This choice is motivated by the fact that the emphasis is put on the definition of admissible evolutions for contacting bodies where the time integration has to be performed. In the rest of the paper, a "minus" sign will always precede the relative tangential velocity $-\dot{\mathbf{u}}_{\mathrm{t}}$ to emphasize its opposite direction to the friction force.

Classically, a rate independent dry friction law is characterized by a kinematic slip rule. In this work, the classical Coulomb friction rule is used. The set of admissible forces, denoted by $K_{\mu}$, is defined by 


$$
K_{\mu}=\left\{\mathbf{r} \in \mathbb{R}^{3} \text { such that }\left\|\mathbf{r}_{\mathrm{t}}\right\|-\mu r_{n} \leqslant 0\right\}
$$

$K_{\mu}$ is the so-called Coulomb's cone and is convex.

\subsection{Complete frictional contact law}

We consider now the previous friction law embedding an impenetrability condition for completeness. On the contact surface $\Gamma_{\mathrm{c}}$, the sliding rule can be combined with the rate form of the Signorini conditions to obtain the frictional contact law that specifies possible scenarios on the contact area (stick, slip and separation). The multivalued nature of this strongly non-linear law makes problems involving frictional contact among the most difficult ones in solid mechanics. Two overlapped "if...then...else" statements can be used to write it analytically:

$$
\begin{array}{|lll|}
\hline \text { if } & r_{n}=0 \text { then } \dot{u}_{n}>0 & \text { ! separating } \\
\text { elseif } & \mathbf{r} \in \text { int } K_{\mu} \text { then } \dot{u}_{n}=0 \text { and } \dot{\mathbf{u}}_{\mathrm{t}}=\mathbf{0} & \text { ! sticking } \\
\text { else } & \left(\mathbf{r} \in \text { bd } K_{\mu} \text { and } r_{n}>0\right) & \\
& \left\{\dot{u}_{n}=0 \text { and } \exists \dot{\lambda}>0 \text { such that }-\dot{\mathbf{u}}_{\mathrm{t}}=\dot{\lambda} \frac{\mathbf{r}_{\mathrm{t}}}{\left\|\mathbf{r}_{\mathrm{t}}\right\|}\right\} & \text { ! sliding } \\
\text { endif } & & \\
\hline
\end{array}
$$

where "int $K_{\mu}$ " and "bd $K_{\mu}$ " denote the interior and the boundary of $K_{\mu}$, respectively. The multivalued character of the law lies in the first and the second part of the statement. If $r_{n}$ is null then $\dot{\mathbf{u}}$ is arbitrary but its normal component $\dot{u}_{n}$ should be positive. In other words, one single element of $\mathbb{R}^{3}(\mathbf{r}=\mathbf{0})$ is associated with an infinite number of velocity vectors $\dot{\mathbf{u}} \in \mathbb{R}^{3}$. The same arguments can be developed for the second part of the statement. The inverse law, i.e. the relationship $\mathbf{r}(-\dot{\mathbf{u}})$, can be written as

\begin{tabular}{|lll|}
\hline if & $\dot{u}_{n}>0$ then $r_{n}=0$ & ! separating \\
elseif & $\dot{\mathbf{u}}=\mathbf{0}$ then $\mathbf{r} \in K_{\mu}$ & ! sticking \\
else & $(\dot{\mathbf{u}} \in \mathbf{T}-\{\mathbf{0}\})$ & \\
& $\left\{\dot{u}_{n}=0\right.$ and $\left.\mathbf{r}_{\mathrm{t}}=\mu r_{n} \frac{-\dot{\mathbf{u}}_{\mathrm{t}}}{\left\|-\dot{\mathbf{u}}_{\mathrm{t}}\right\|}\right\}$ & ! sliding \\
endif & & \\
\hline
\end{tabular}

The complete form of the frictional contact law involves three possible states, which are separating, contact with sticking, and contact with sliding. Only the last state produces energy dissipation.

\section{The bi-potential method}

De Saxcé and Feng [5] have shown that the contact law (13) is equivalent to the following differential inclusion:

$$
\left(-\dot{\mathbf{u}}_{\mathrm{t}}-\left(\dot{u}_{n}+\mu\left\|-\dot{\mathbf{u}}_{\mathrm{t}}\right\|\right) \mathbf{n}\right) \in \partial \bigcup_{K_{\mu}} \mathbf{r}
$$

where $\bigcup_{K_{\mu}} \mathbf{r}$ denotes the so-called indicator function of the closed convex set $K_{\mu}$ :

$$
\bigcup_{K_{\mu}}(\mathbf{r})= \begin{cases}0 & \text { if } \mathbf{r} \in K_{\mu} \\ +\infty & \text { otherwise }\end{cases}
$$

The following contact bi-potential is obtained:

$$
b_{\mathrm{c}}(-\dot{\mathbf{u}}, \mathbf{r})=\bigcup_{\mathbb{R}_{-}}\left(-\dot{u}_{n}\right)+\bigcup_{K_{\mu}}(\mathbf{r})+\mu r_{n}\left\|-\dot{\mathbf{u}}_{\mathrm{t}}\right\|
$$

where $\left.\left.\mathbb{R}_{-}=\right]-\infty, 0\right]$ is the set of the negative and null real numbers. 
In order to avoid non-differentiable potentials that occur in non-linear mechanics, such as in contact problems, it is convenient to use the augmented Lagrangian method [5]. For the contact bi-potential $b_{\mathrm{c}}$, given by (17), provided that $\dot{u}_{n} \geqslant 0$ and $\mathbf{r} \in K_{\mu}$, we have:

$$
\forall \mathbf{r}^{\prime} \in K_{\mu}, \quad \varrho \mu\left(r_{n}^{\prime}-r_{n}\right)\left\|-\dot{\mathbf{u}}_{\mathrm{t}}\right\|+\left(\mathbf{r}^{\prime}-(\mathbf{r}-\varrho \dot{\mathbf{u}})\right) \cdot\left(\mathbf{r}^{\prime}-\mathbf{r}\right) \geqslant 0
$$

where $\varrho$ is a solution parameter which is not user-defined. From numerical experiments, $\varrho$ can be chosen as the maximum value of the diagonal terms of the local contact stiffness matrix. Taking account of the decomposition (3) and (4), the following inequality has to be satisfied:

$$
\mathbf{r}^{\prime} \in K_{\mu}, \quad(\mathbf{r}-\tau) \cdot\left(\mathbf{r}^{\prime}-\mathbf{r}\right) \geqslant 0
$$

where the modified augmented surface traction $\tau$ is defined by

$$
\tau=\mathbf{r}+\varrho\left(-\dot{\mathbf{u}}_{\mathrm{t}}-\left(\dot{u}_{n}+\mu\left\|-\dot{\mathbf{u}}_{\mathrm{t}}\right\|\right) \mathbf{n}\right)
$$

The inequality (19) means that $\mathbf{r}$ is the projection of $\tau$ onto the closed convex Coulomb's cone:

$$
\mathbf{r}=\operatorname{proj}\left(\tau, K_{\mu}\right)
$$

For the numerical solution of the implicit Eq. (21), Uzawa's algorithm can be used, which leads to an iterative process involving one predictor-corrector step:

$$
\begin{aligned}
& \text { Predictor } \tau^{i+1}=\mathbf{r}^{i}+\varrho^{i}\left(-\dot{\mathbf{u}}_{\mathrm{t}}^{i}-\left(\dot{u}_{n}^{i}+\mu\left\|-\dot{\mathbf{u}}_{\mathrm{t}}^{i}\right\|\right) \mathbf{n}\right) \\
& \text { Corrector } \mathbf{r}^{i+1}=\operatorname{proj}\left(\tau^{i+1}, K_{\mu}\right)
\end{aligned}
$$

It is worth noting that, in this algorithm, the unilateral contact and the friction are coupled via the bi-potential. Another gist of the bi-potential method is that the corrector can be analytically found with respect to the three possible contact statuses: $\tau \subset K_{\mu}$ (contact with sticking), $\tau \subset K_{\mu}^{*}$ (no contact) and $\tau \subset \mathbb{R}^{3}-K_{\mu} \cup K_{\mu}^{*}$ (contact with sliding). $K_{\mu}^{*}$ is the polar cone of $K_{\mu}$. This corrector step is explicitly given as follows:

$$
\begin{array}{|lll|}
\text { if } & \mu\left\|\tau_{t}^{i+1}\right\|<-\tau_{n}^{i+1} \text { then } \mathbf{r}^{i+1}=0 & \text { ! separating } \\
\text { elseif } & \left\|\tau_{t}^{i+1}\right\|<\mu \tau_{n}^{i+1} \text { then } \mathbf{r}^{i+1}=\tau^{i+1} & \text { ! sticking } \\
\text { else } & \mathbf{r}^{i+1}=\tau^{i+1}-\frac{\left(\left\|\tau_{t}^{i+1}\right\|-\mu \tau_{n}^{i+1}\right)}{\left(1+\mu^{2}\right)}\left(\frac{\tau_{t}^{i+1}}{\left\|\tau_{\mathrm{t}}^{i+1}\right\|}+\mu \mathbf{n}\right) & \text { ! sliding } \\
\hline
\end{array}
$$

It is important to emphasize the fact that this explicit formula is valid for both $2 \mathrm{D}$ and $3 \mathrm{D}$ contact problems with Coulomb's friction and allows us to obtain very stable and accurate results.

\section{Hyperelastic bodies undergoing large deformations}

Rubber or other polymer materials are said to be hyperelastic. Usually, these kind of materials undergo large deformations. In order to describe the geometrical transformation problems, the deformation gradient tensor is introduced by

$$
F_{i j}(\mathbf{x})=\delta_{i j}+\frac{\partial u_{i}}{\partial x_{j}} \text { or } \mathbf{F}=\mathbf{I}+\nabla \mathbf{u}
$$

where $\mathbf{I}$ is the unity tensor, $\mathbf{x}$ the position vector and $\mathbf{u}$ the displacement vector. Because of large displacements and rotations, Green-Lagrangian strain is adopted for the non-linear relationships between strains and displacements. We note $\mathbf{C}$ the stretch tensor or the right Cauchy-Green deformation tensor $\left(\mathbf{C}=\mathbf{F}^{\mathrm{T}} \mathbf{F}\right)$. The Green-Lagrangian strain tensor $\mathbf{E}$ is defined by

$$
\mathbf{E}=(\mathbf{C}-\mathbf{I}) / 2
$$

In the case of hyperelastic law, there exists an elastic potential function $W$ (or strain energy density function) which is a scale function of one of the strain tensors, whose derivative with respect to a strain component determines the corresponding stress component. This can be expressed by

$$
\mathbf{S}=\frac{\partial W}{\partial \mathbf{E}}=2 \frac{\partial W}{\partial \mathbf{C}}
$$


where $\mathbf{S}$ is the second Piola-Kirchoff stress tensor. In the particular case of isotropic hyperelasticity [16], (26) can be written by

$$
\mathbf{S}=2\left[I_{3} \frac{\partial W}{\partial I_{3}} \mathbf{C}^{-1}+\left(\frac{\partial W}{\partial I_{1}}+I_{1} \frac{\partial W}{\partial I_{2}}\right) \mathbf{I}-\frac{\partial W}{\partial I_{2}} \mathbf{C}\right]
$$

where $I_{i}(i=1,2,3)$ denote the invariants of the right Cauchy-Green deformation tensor $\mathbf{C}$ :

$$
I_{1}=C_{i i} ; \quad I_{2}=\left(I_{1}^{2}-C_{i j} C_{i j}\right) / 2 ; \quad I_{3}=\operatorname{det}(\mathbf{C})
$$

The Blatz-Ko constitutive law is used to model compressible foam-type polyurethane rubbers [17]. The strain energy density function is given as follows:

$$
W=\frac{G}{2}\left[\frac{I_{2}}{I_{3}}+2 \sqrt{I_{3}}-5\right]
$$

where $G$ is the shear modulus. By deriving the energy density (29) with respect to the three invariants, we obtain

$$
\frac{\partial W}{\partial I_{1}}=0 ; \quad \frac{\partial W}{\partial I_{2}}=\frac{G}{2} \frac{1}{I_{3}} ; \quad \frac{\partial W}{\partial I_{3}}=\frac{G}{2}\left[-\frac{I_{2}}{I_{3}^{2}}+\frac{1}{\sqrt{I_{3}}}\right]
$$

Reporting the result in (27) gives

$$
\mathbf{S}=G \mathbf{F}^{-1}\left\{\sqrt{I_{3}} \mathbf{I}-\mathbf{B}^{-1}\right\} \mathbf{F}^{-T}
$$

where $\mathbf{B}=\mathbf{F F}^{\mathrm{T}}$ is the left Cauchy-Green deformation tensor associated to $\mathbf{F}$. Noting $J=\sqrt{\operatorname{det}(2 \mathbf{E}+\mathbf{I})}$, the tensor $\mathbf{S}$ can also be written in function of $\mathbf{E}$ :

$$
\mathbf{S}(\mathbf{E})=G\left\{J(2 \mathbf{E}+\mathbf{I})^{-1}-(2 \mathbf{E}+\mathbf{I})^{-2}\right\}
$$

The Cauchy stress (or true stress) tensor $\boldsymbol{\sigma}$ is calculated from the second Piola-Kirchoff stress tensor $\mathbf{S}$ as follows:

$$
\boldsymbol{\sigma}=\frac{1}{\operatorname{det}(\mathbf{F})} \mathbf{F S F}^{T}
$$

\section{Finite element formulation of non-linear structures}

\subsection{Total Lagrangian formulation}

In the case of dynamic multibody contact problems involving large deformations of hyperelastic solids, the non-linear relation between strains and displacements cannot be ignored. The total Lagrangian formulation was selected in this work to describe non-linear behavior. In the context of the finite element method and with (24) and (25), the Green-Lagrangian strain can be formally written with linear and non-linear terms in function of nodal displacements:

$$
\mathbf{E}=\left(\mathbf{B}_{\mathrm{L}}+\frac{1}{2} \mathbf{B}_{\mathrm{NL}}(\mathbf{u})\right) \mathbf{u}
$$

where $\mathbf{B}_{\mathrm{L}}$ is the matrix which relates the linear strain term to the nodal displacements, and $\mathbf{B}_{\mathrm{NL}}(\mathbf{u})$, the matrix which relates the non-linear strain term to the nodal displacements. From Eq. (34), the incremental form of the strain-displacement relationship is

$$
\delta \mathbf{E}=\left(\mathbf{B}_{\mathrm{L}}+\mathbf{B}_{\mathrm{NL}}(\mathbf{u})\right) \delta \mathbf{u}
$$

Using the principle of virtual displacement, the virtual work $\delta U$ is given as

$$
\delta U=\mathbf{M} \ddot{\mathbf{u}} \delta \mathbf{u}+\mathbf{A} \dot{\mathbf{u}} \delta \mathbf{u}+\int_{V_{0}} \mathbf{S} \delta \mathbf{E} \mathrm{d} V-\mathbf{F}_{\text {ext }} \delta \mathbf{u}-\mathbf{R} \delta \mathbf{u}=0
$$


where $V_{0}$ is the volume of the initial configuration, $\mathbf{F}_{\text {ext }}$ the vector of external loads, $\mathbf{R}$ the contact reaction vector, $\mathbf{M}$ the mass matrix, A the damping matrix, $\dot{\mathbf{u}}$ the velocity vector and $\ddot{\mathbf{u}}$ the acceleration vector.

From Eqs. (26)-(31), we obtain

$$
\delta \mathbf{S}=\mathbf{D} \delta \mathbf{E}=\mathbf{D}\left(\mathbf{B}_{\mathrm{L}}+\mathbf{B}_{\mathrm{NL}}(\mathbf{u})\right) \delta \mathbf{u}
$$

where $\mathbf{D}$ is the current stress-strain tensor which is obtained from the derivative of $\mathbf{S}$ with respect to $\mathbf{E}$ in Eq. (32):

$$
\begin{aligned}
D_{i j k l}= & G\left\{-2 J(2 \mathbf{E}+\mathbf{I})_{i k}^{-1}(2 \mathbf{E}+\mathbf{I})_{l j}^{-1}+J(2 \mathbf{E}+\mathbf{I})_{l k}^{-1}(2 \mathbf{E}+\mathbf{I})_{i j}^{-1}+2\left[(2 \mathbf{E}+\mathbf{I})_{i k}^{-1}(2 \mathbf{E}+\mathbf{I})_{l j}^{-2}\right.\right. \\
& \left.\left.+(2 \mathbf{E}+\mathbf{I})_{i k}^{-2}(2 \mathbf{E}+\mathbf{I})_{l j}^{-1}\right]\right\}
\end{aligned}
$$

Substituting $\delta \mathbf{E}$ from Eq. (35) into Eq. (36) results in

$$
\delta U=\mathbf{M} \ddot{\mathbf{u}} \delta \mathbf{u}+\mathbf{A} \dot{\mathbf{u}} \delta \mathbf{u}+\int_{V_{0}} \mathbf{S}\left(\mathbf{B}_{\mathrm{L}}+\mathbf{B}_{\mathrm{NL}}(\mathbf{u})\right) \delta \mathbf{u} \mathrm{d} V-\mathbf{F}_{\mathrm{ext}} \delta \mathbf{u}-\mathbf{R} \delta \mathbf{u}=0
$$

The vector of internal forces is defined by

$$
\mathbf{F}_{\text {int }}=\int_{V_{0}}\left(\mathbf{B}_{\mathrm{L}}+\mathbf{B}_{\mathrm{NL}}(\mathbf{u})\right)^{\mathrm{T}} \mathbf{S} \mathrm{d} V
$$

Since $\delta \mathbf{u}$ is arbitrary, a set of non-linear equations can be obtained as

$$
\mathbf{M u ̈}+\mathbf{A} \dot{\mathbf{u}}+\mathbf{F}_{\text {int }}-\mathbf{F}_{\text {ext }}-\mathbf{R}=0
$$

It is noted that the stiffness effect is taken into account by the internal forces vector $\mathbf{F}_{\text {int }}$. Eq. (41) can be transformed into

$$
\mathbf{M u ̈}=\mathbf{F}+\mathbf{R}, \quad \text { where } \mathbf{F}=\mathbf{F}_{\mathrm{ext}}-\mathbf{F}_{\mathrm{int}}-\mathbf{A} \dot{\mathbf{u}}
$$

with the initial conditions at $t=0$

$$
\dot{\mathbf{u}}=\dot{\mathbf{u}}_{0} \quad \text { and } \quad \mathbf{u}=\mathbf{u}_{0}
$$

Taking the derivative of $\mathbf{F}_{\text {int }}$ with respect to the nodal displacements $\mathbf{u}$ gives the tangent stiffness matrix as

$$
\mathbf{K}=\frac{\partial \mathbf{F}_{\text {int }}}{\partial \mathbf{u}}=\int_{V_{0}}\left(\frac{\partial \mathbf{S}}{\partial \mathbf{u}}\left(\mathbf{B}_{\mathrm{L}}+\mathbf{B}_{\mathrm{NL}}(\mathbf{u})\right)+\mathbf{S} \frac{\partial \mathbf{B}_{\mathrm{NL}}(\mathbf{u})}{\partial \mathbf{u}}\right) \mathrm{d} V
$$

In addition, by using Eqs. (35) and (37), the tangent stiffness matrix is in fact the sum of the elastic stiffness matrix $\mathbf{K}_{\mathrm{e}}$, the geometric stiffness (or initial stress stiffness) matrix $\mathbf{K}_{\sigma}$ and the initial displacement stiffness matrix $\mathbf{K}_{\mathrm{u}}$ :

$$
\mathbf{K}=\mathbf{K}_{\mathrm{e}}+\mathbf{K}_{\sigma}+\mathbf{K}_{\mathrm{u}}
$$

where

$$
\begin{aligned}
& \mathbf{K}_{\mathrm{e}}=\int_{V_{0}} \mathbf{B}_{\mathrm{L}}^{\mathrm{T}} \mathbf{D} \mathbf{B}_{\mathrm{L}} \mathrm{d} V \\
& \mathbf{K}_{\sigma}=\int_{V_{0}} \mathbf{S} \frac{\partial \mathbf{B}_{\mathrm{NL}}}{\partial \mathbf{u}} \mathrm{d} V \\
& \mathbf{K}_{\mathrm{u}}=\int_{V_{0}}\left(\mathbf{B}_{\mathrm{L}}^{\mathrm{T}} \mathbf{D} \mathbf{B}_{\mathrm{NL}}+\mathbf{B}_{\mathrm{NL}}^{\mathrm{T}} \mathbf{D} \mathbf{B}_{L}+\mathbf{B}_{\mathrm{NL}}^{\mathrm{T}} \mathbf{D} \mathbf{B}_{\mathrm{NL}}\right) \mathrm{d} V
\end{aligned}
$$

\subsection{First order integration algorithm}

We can now integrate Eq. (42) between consecutive time configuration $t$ and $t+\Delta t$. The most common method to do that is the Newmark method which is based on a second order algorithm. However, in impact 
problems, higher order approximation does not necessarily mean better accuracy, and may even be superfluous. At the moment of a sudden change of contact conditions (impact, release of contact), the velocity and acceleration are not continuous, and excessive regularity constraints may lead to serious errors. For this reason, Jean [15] has proposed a first order algorithm which is used in this work. This algorithm is based on the following approximations:

$$
\begin{aligned}
& \int_{t}^{t+\Delta t} \mathbf{M} \mathrm{d} \dot{\mathbf{u}}=\mathbf{M}\left(\dot{\mathbf{u}}^{t+\Delta t}-\dot{\mathbf{u}}^{t}\right) \\
& \int_{t}^{t+\Delta t} \mathbf{F} \mathrm{d} t=\Delta t\left((1-\xi) \mathbf{F}^{t}+\xi \mathbf{F}^{t+\Delta t}\right) \\
& \int_{t}^{t+\Delta t} \mathbf{R} \mathrm{d} t=\Delta t \mathbf{R}^{t+\Delta t} \\
& \mathbf{u}^{t+\Delta t}-\mathbf{u}^{t}=\Delta t\left((1-\theta) \dot{\mathbf{u}}^{t}+\theta \dot{\mathbf{u}}^{t+\Delta t}\right)
\end{aligned}
$$

where $0 \leqslant \xi \leqslant 1 ; 0 \leqslant \theta \leqslant 1$. In the iterative solution procedure, all the values at time $t+\Delta t$ are replaced by the values of the current iteration $i+1$; for example, $\mathbf{F}^{t+\Delta t}=\mathbf{F}^{i+1}$. A standard approximation of $\mathbf{F}^{i+1}$ gives

$$
\mathbf{F}^{i+1}=\mathbf{F}_{\text {int }}^{i}+\frac{\partial \mathbf{F}}{\partial \mathbf{u}}\left(\mathbf{u}^{i+1}-\mathbf{u}^{i}\right)+\frac{\partial \mathbf{F}}{\partial \dot{\mathbf{u}}}\left(\dot{\mathbf{u}}^{i+1}-\dot{\mathbf{u}}^{i}\right)=\mathbf{F}_{\text {int }}^{i}-\mathbf{K}^{i} \Delta \mathbf{u}-\mathbf{A}^{i} \Delta \dot{\mathbf{u}}
$$

Finally, we obtain the recursive form of (42) in terms of displacements:

$$
\begin{aligned}
& \overline{\mathbf{K}}^{i} \Delta \mathbf{u}=\overline{\mathbf{F}}^{i}+\overline{\mathbf{F}}_{\mathrm{acc}}^{i}+\mathbf{R}^{i+1} \\
& \mathbf{u}^{i+1}=\mathbf{u}^{i}+\Delta \mathbf{u}
\end{aligned}
$$

where the so-called effective terms are given by

$$
\begin{aligned}
& \overline{\mathbf{K}}^{i}=\xi \mathbf{K}^{i}+\frac{\xi}{\theta \Delta t} \mathbf{A}^{i}+\frac{1}{\theta \Delta t^{2}} \mathbf{M}^{i} \\
& \overline{\mathbf{F}}_{\mathrm{acc}}^{i}=-\frac{1}{\theta \Delta t^{2}} \mathbf{M}^{i}\left\{\mathbf{u}^{i}-\mathbf{u}^{t}-\Delta t \dot{\mathbf{u}}^{t}\right\} \\
& \overline{\mathbf{F}}^{i}=(1-\xi)\left(\mathbf{F}_{\mathrm{int}}^{t}+\mathbf{F}_{\mathrm{ext}}^{t}\right)+\xi\left(\mathbf{F}_{\mathrm{int}}^{i}+\mathbf{F}_{\mathrm{ext}}^{t+\Delta t}\right)
\end{aligned}
$$

At the end of each time step, the velocity is updated by

$$
\dot{\mathbf{u}}^{t+\Delta t}=\left(1-\frac{1}{\theta}\right) \dot{\mathbf{u}}^{t}+\frac{1}{\theta \Delta t}\left(\mathbf{u}^{t+\Delta t}-\mathbf{u}^{t}\right)
$$

By setting $\theta=\frac{1}{2}$, this scheme is then called the implicit trapezoidal rule and it is equivalent to the TammaNamburu method in which the acceleration need not be computed [18]. See [19,20] for the interesting comments on time stepping algorithms and on energy conservation.

It is noted that Eq. (54) is strongly non-linear, because of large rotations and large displacements of solid, for instance in multibody contact/impact problems. Besides, as mentioned above, the constitutive law of contact with friction is usually represented by inequalities and the contact potential is even non-differentiable. Instead of solving this equation in consideration of all non-linearities at the same time, Feng [21] has proposed a solution strategy which consists in separating the non-linearities in order to overcome the complexity of calculation and to improve the numerical stability. As $\Delta \mathbf{u}$ and $\mathbf{R}$ are both unknown, Eq. (54) cannot be directly solved. First, the vector $\mathbf{R}$ is determined by the bi-potential method in a reduced system, which only concerns contact nodes. Then, the vector $\Delta \mathbf{u}$ can be computed in the whole structure, using contact reactions as external loading. It is very important to note that, as opposed to the penalty method or Lagrange multiplier method, the bi-potential method neither changes the global stiffness matrix, nor increases the degrees of freedom. One consequence of this interesting property is that it is easy to implement contact and friction problems in an existing general-purpose finite element code by this method. In addition, the solution procedure is more stable because of the separation of non-linearities and improved numerical algorithms for calculation of contact reactions. 


\subsection{Energy computation}

After determining the displacement and the velocity fields, we can calculate different energies. The total elastic strain energy of the contact bodies (discretized by $n_{\mathrm{el}}$ finite elements) is then written by

$$
E_{\mathrm{e}}=\sum_{e=1}^{n_{\mathrm{el}}} \int_{\Omega_{\mathrm{e}}} W_{\mathrm{e}} \mathrm{d} \Omega
$$

The total kinetic energy can be calculated at the global level by

$$
E_{\mathrm{k}}=\frac{1}{2} \dot{\mathbf{u}}^{\mathrm{T}} \mathbf{M} \dot{\mathbf{u}}
$$

Finally, the total energy of the system of solids is

$$
E_{\mathrm{t}}=E_{\mathrm{e}}+E_{\mathrm{k}}
$$

The case of interest for the analysis presented below corresponds to the homogeneous Neumann problem, characterized by no imposed boundary displacements and no external loading. In addition, if frictionless contact is considered, the total energy should be conserved. For the given examples, this fundamental energy conservation property has been observed.

\section{Numerical results}

The algorithms presented above have been implemented and tested in the finite element code FER/impact [22]. Many application examples, in static or quasi-static cases, have been carried out using the present method $[1,21,23]$.

To illustrate the behavior of a contact/impact simulation by the new algorithm described above, we consider two example applications. For each cases, we assume that no damping exists except for Coulomb friction between contact surfaces, i.e. $\mathbf{A}=0$ in Eqs. (41), (42) and (55).

\subsection{Deformable-rigid impact}

This problem concerns the impact of a cylinder made of foam-type polyurethane rubbers into two oblique rigid symmetric surfaces forming a funnel. The characteristics of this example are: shear modulus $G=3 \mathrm{MPa}$, mass density $\rho=700 \mathrm{~kg} / \mathrm{m}^{3}$, initial velocity $v_{y}=-30 \mathrm{~m} / \mathrm{s}$. The radius of the cylinder is: $R=0.01 \mathrm{~m}$. The total simulation time is $3 \times 10^{-3} \mathrm{~s}$ and the solution parameters are: $\Delta t=10^{-5} \mathrm{~s}, \xi=\theta=0.5$. The cylinder is modeled by 209 nodes and 192 linear quadrilateral plane strain elements (Fig. 2). The initial position of the

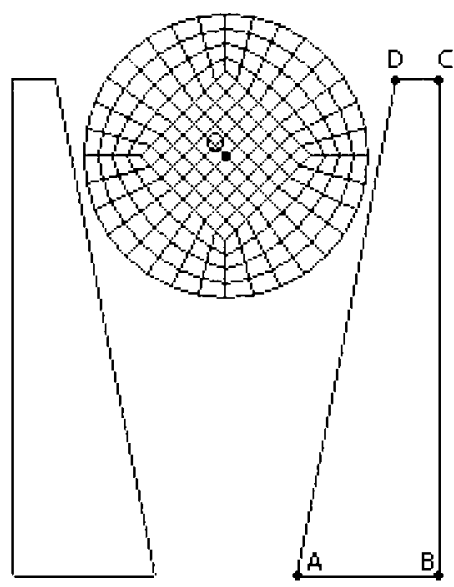

Fig. 2. Deformable-rigid impact. 
cylinder is given by its center point $\mathrm{O}(0.0,0.03)$. The right part of the rigid block is defined by $A(0.005,0.0)$, $B(0.015,0.0), C(0.015,0.035)$ and $D(0.012,0.035)$.

Three cases A, B and C are considered (Table 1). It is noted that these analyses were performed on a PC (Pentium 4/2.8 GHz). Fig. 3 shows the Von-Mises stress distribution in the cylinder at the moment when the cylinder reaches its lowest position and the kinetic energy $E_{\mathrm{k}}$ equals zero (see Figs. 6-8). The CPU time to achieve the solution and the maximum value of the Von-Mises stress are also given in Table 1. We observe apparent differences concerning the values and the localization of the maximum stress. In Case A, the cylinder goes down lower so as to be more deformed. Therefore, the stress value is higher. When the friction increases, the cylinder does not go down as low, braked by the friction forces. The localization of the maximum stress moves to the contact surfaces because friction forces are added onto the surfaces in contact, as shown in Fig. 3. The deformed configurations at time $t=2 \mathrm{~ms}$ are shown in Fig. 4. The displacement of the center point $\mathrm{O}$

Table 1

Influence of friction coefficients

\begin{tabular}{llll}
\hline Case & At time $(\mathrm{ms})$ & $\sigma_{\max }(\mathrm{MPa})$ & CPU time $(\mathrm{s})$ \\
\hline A: $\mu=0.0$ & 0.87 & 8.192 & 62 \\
B: $\mu=0.2$ & 0.70 & 4.523 & 77 \\
C: $\mu=0.4$ & 0.61 & 4.396 & 83 \\
\hline
\end{tabular}

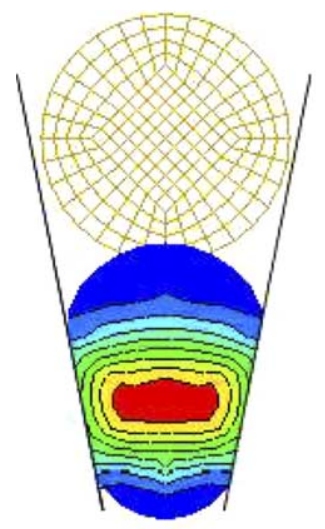

A

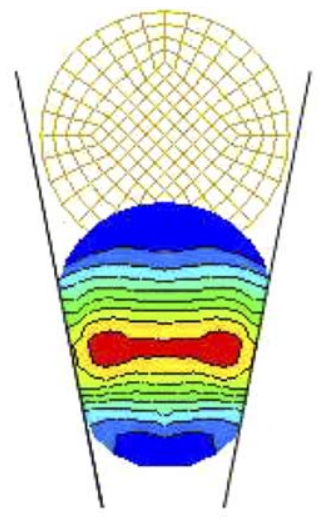

B

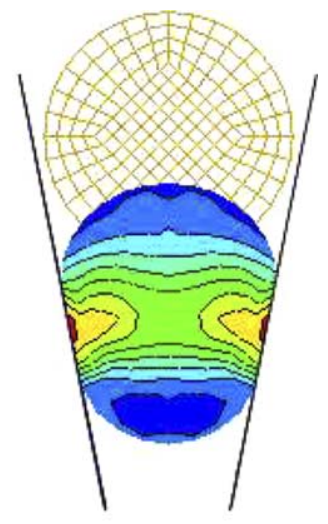

C

Fig. 3. Isovalues of Von-Mises stress.
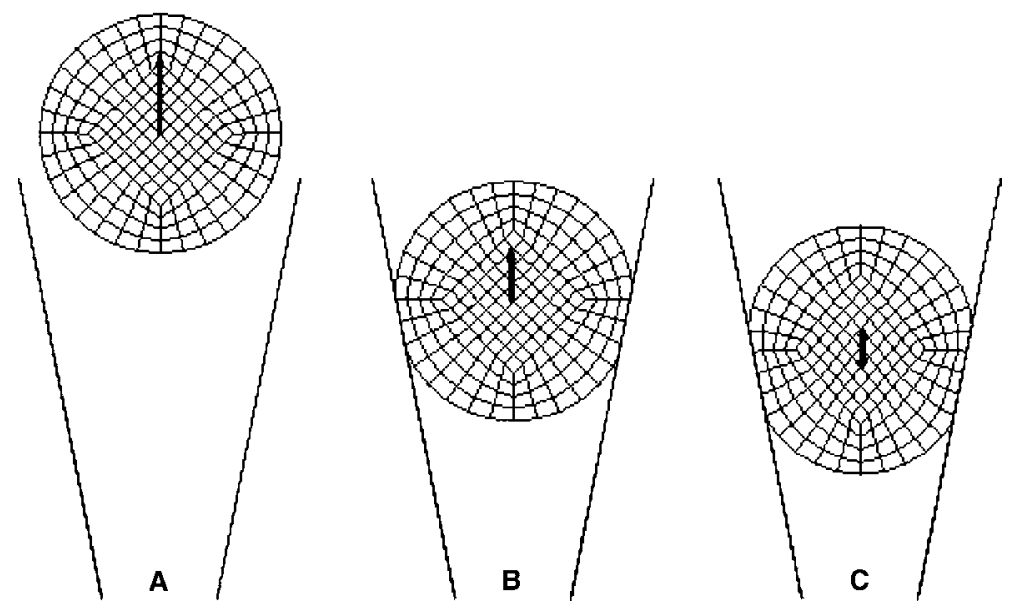

Fig. 4. Deformed configurations at time $t=2 \mathrm{~ms}$. 


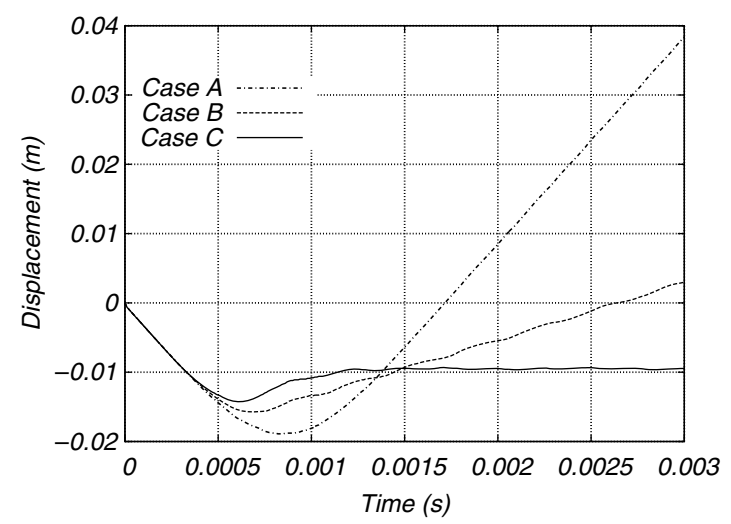

Fig. 5. Displacement of point $O$.

versus time is plotted in Fig. 5. For the three cases, Figs. 6-8 show the plots of the kinetic energy $E_{\mathrm{k}}$, the elastic strain energy $E_{\mathrm{e}}$ and the total energy $E_{\mathrm{t}}$. For Case A, the cylinder rebounds with the same velocity as the initial velocity, as there is no energy loss in the system (see Fig. 6). For Case B, the cylinder rebounds too but with smaller velocity than the initial velocity, as there is energy loss in the system (see Fig. 7). By taking into account more important friction (Case C), after a jump up, the cylinder sticks to the contact surfaces and

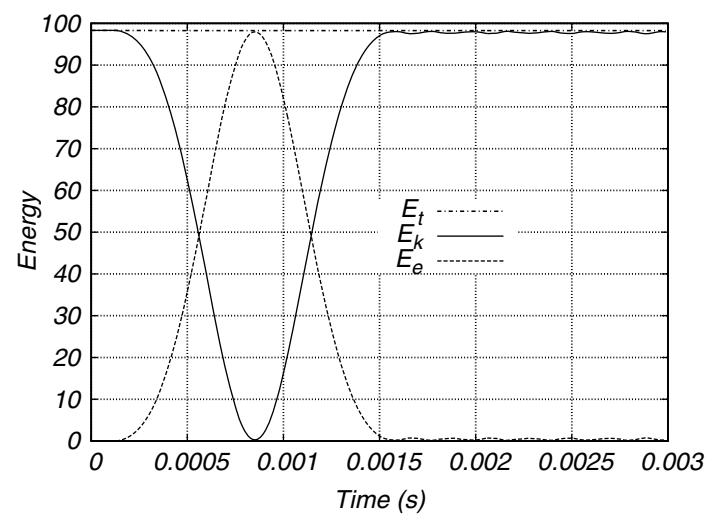

Fig. 6. Energy evolution $(\mu=0.0)$.

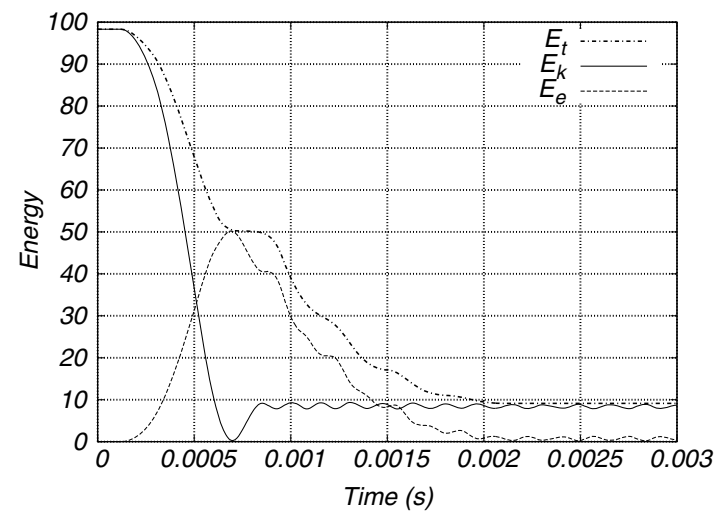

Fig. 7. Energy evolution $(\mu=0.2)$. 


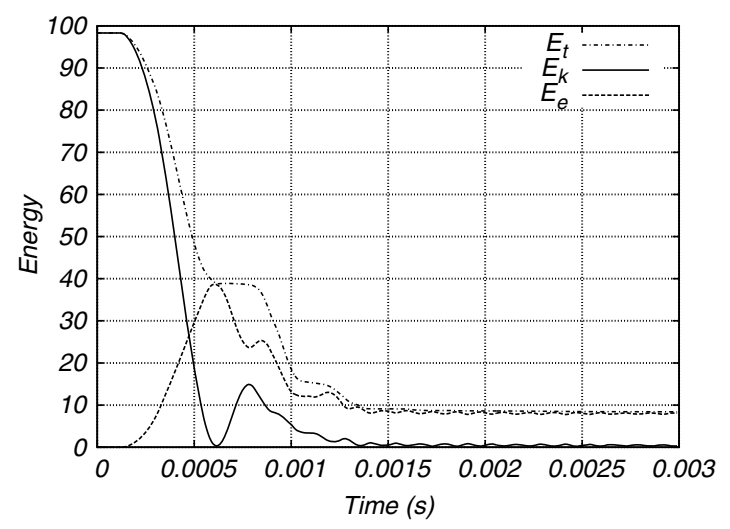

Fig. 8. Energy evolution $(\mu=0.4)$.

the kinetic energy $E_{\mathrm{k}}$ tends to zero and thus the cylinder is locked (see Fig. 8). Furthermore, the elastic deformation is smaller, as compared to the frictionless case A.

We observe that the total energy is perfectly conserved in the case of frictionless contact (Fig. 6). However, in the case of frictional contact, the total energy decreases (Figs. 7 and 8). So the total energy is dissipated by frictional effects as expected. It is worth noting that the dissipated energy is quantitatively calculated. It is also interesting to examine another question: is the dissipated energy proportional to the friction coefficient? The answer is not according to numerical results. The proof is illustrated by Figs. 7 and 8 in which we observe almost the same dissipated energy even with two different friction coefficients. In fact, when the friction coefficient increases, the friction forces increase. However, the tangential slips will decrease (Fig. 3). We know that the dissipated energy depends not only on the friction forces but also on the tangential slips on the contact surface.

We observed another interesting result in this study when comparing the cases B and C. In Case B (Fig. 7), the kinetic energy tends to a constant value and the elastic strain energy equals zero after the impact. On the contrary, in Case $\mathrm{C}$ (Fig. 8), the kinetic energy tends to zero and the elastic strain energy is not released. As expected, the cylinder is locked inside the funnel. For both cases, the total energy remains almost the same.

\subsection{Deformable-deformable-rigid impact}

In order to show the ability of the algorithms developed to deal with multi-zones contact/impact problems, we propose a second example which simulates the impact of a deformable cylinder onto a deformable block and the later is posed onto a rigid foundation. So the contact occurs not only between the cylinder and the block but also between the block and the rigid foundation. The material characteristics of the two hyperelastic bodies are: shear modulus $G=3 \mathrm{MPa}$, mass density $\rho=700 \mathrm{~kg} / \mathrm{m}^{3}$. The initial velocity of the cylinder is:

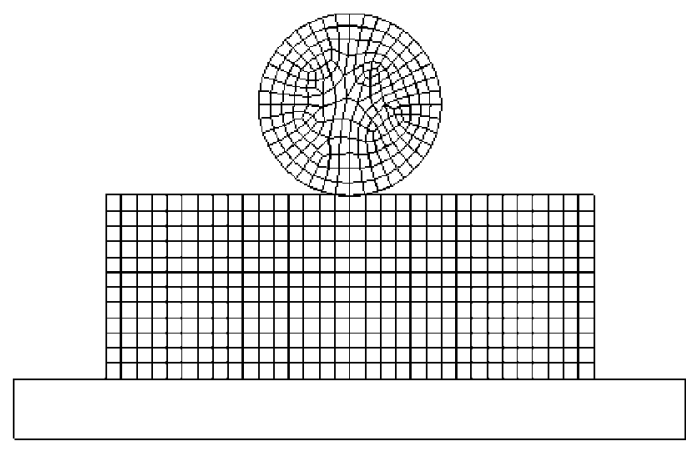

Fig. 9. Deformable-deformable-rigid impact. 


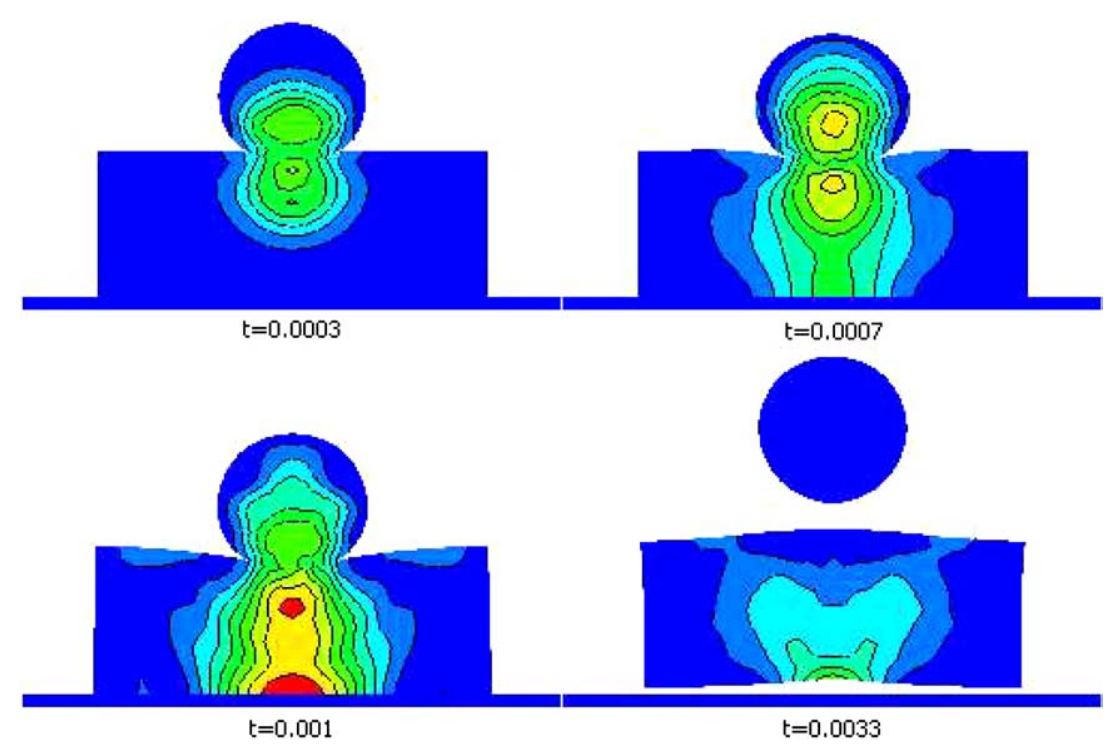

Fig. 10. Isovalues of Von-Mises stress.

$v_{y}=-20 \mathrm{~m} / \mathrm{s}$. The radius of the cylinder is: $R=0.025 \mathrm{~m}$. The geometric sizes of the block are: height $l=0.06 \mathrm{~m}$ and width $L=0.16 \mathrm{~m}$. The total simulation time is $5 \times 10^{-3} \mathrm{~s}$ and the solution parameters are: $\Delta t=10^{-5} \mathrm{~s}, \xi=\theta=0.5$. The cylinder and the block are modeled respectively by 239 and 429 nodes for 384 and 118 linear quadrilateral plane strain elements (Fig. 9).

Fig. 10 shows the distribution of the Von-Mises stress in the two deformable bodies during the simulation. It is interesting to note that the block jumps up under the impact of the cylinder.

\section{Conclusion}

In this paper, we have presented the recent development of the bi-potential method applied to dynamic analysis of two-dimensional contact problems with Coulomb friction between Blatz-Ko hyperelastic bodies. The algorithm has been described and investigated numerically for two problems using different coefficients of friction. From numerical experiments, we have found that:

- The algorithm is simple and efficient:

- no modification of the global stiffness matrix;

- no regularization of contact and friction laws;

- accurate calculation of contact forces in a reduced system;

- first order time stepping instead of second or higher order integration.

- The total energy is well conserved in the case of frictionless contact of solids.

- The algorithm permits to determine quantitatively the physical energy dissipation by frictional effects.

- The locking and jumping phenomena are numerically illustrated.

We have felt that this approach could easily be extended to three-dimensional dynamic contact problems including non-linear material constitutive laws and more complex frictional models [24]. This work is being undertaken.

\section{References}

[1] Z.Q. Feng, F. Peyraut, N. Labed, Solution of large deformation contact problems with friction between Blatz-Ko hyperelastic bodies, Int. J. Eng. Sci. 41 (2003) 2213-2225. 
[2] N. Kikuchi, J.T. Oden, Contact Problems in Elasticity: a Study of Variational Inequalities and Finite Elements, SIAM, Philadelphia, 1988.

[3] Z.H. Zhong, Finite Element Procedures in Contact-Impact Problems, Oxford University Press, Oxford, 1993.

[4] P. Wriggers, Computational Contact Mechanics, John Wiley \& Sons, 2002.

[5] G. De Saxcé, Z.Q. Feng, The bi-potential method: a constructive approach to design the complete contact law with friction and improved numerical algorithms, Math. Comput. Model. 28 (4-8) (1998) 225-245 (Special issue, Recent Advances in Contact Mechanics).

[6] H.M. Hilber, T.J.R. Hughes, R.L. Taylor, Improved numerical dissipation for the time integration algorithms in structural dynamics, Earthquake Eng. Struct. Dyn. 5 (1977) 283-292.

[7] T.J.R. Hughes, R.L. Taylor, J.L. Sackman, A. Curnier, W. Kanoknukulchai, A finite element method for a class of contact-impact problems, Comput. Methods Appl. Mech. Eng. 8 (1976) 249-276.

[8] P. Wriggers, T. Vu Van, E. Stein, Finite element formulation of large deformation impact contact problems with friction, Comput. Struct. 37 (1990) 319-331.

[9] F. Armero, E. Petocz, Formulation and analysis of conserving algorithms for frictionless dynamic contact/impact problems, Comput. Methods Appl. Mech. Eng. 158 (1998) 269-300.

[10] F. Armero, E. Petocz, A new dissipative time-stepping algorithm for frictional contact problems: formulation and analysis, Comput. Methods Appl. Mech. Eng. 179 (1999) 151-178.

[11] T.A. Laursen, V. Chawla, Design of energy conserving algorithms for frictionless dynamic contact problems, Int. J. Numer. Methods Eng. 40 (1997) 863-886.

[12] T.A. Laursen, G.R. Love, Improved implicit integrators for transient impact problems geometric admissibility within the conserving framework, Int. J. Numer. Meth. Eng. 53 (2002) 245-274.

[13] G.R. Love, T.A. Laursen, Improved implicit integrators for transient impact problems dynamic frictional dissipation within an admissible conserving framework, Comput. Methods Appl. Mech. Eng. 192 (2003) 2223-2248.

[14] O.C. Zienkiewicz, W.L. Wood, L.W. Hine, R.L. Taylor, A unified set of single step algorithms. Part 1. General formulation and application, Int. J. Numer. Methods Eng. 20 (1984) 1529-1552.

[15] M. Jean, Dynamics with partially elastic shocks and dry friction: double scale method and numerical approach, in: Proceedings of the 4th Meeting on unilateral problems in structural analysis, Capri, 1989.

[16] P.G. Ciarlet, Elasticité Tridimensionnelle, Masson Collection RMA, 1985.

[17] P.J. Blatz, W.L. Ko, Application of finite elastic theory to the deformation of rubbery materials, Trans. Soc. Rheol. 6 (1962) $223-251$.

[18] T.K. Kamma, R.R. Namburu, A robust self-starting explicit computational methodology for structural dynamic applications: architecture and representations, Int. J. Numer. Methods Eng. 30 (1990) 1441-1454.

[19] J.C. Simo, K.K. Wong, Unconditionally stable algorithms for rigid body dynamics that exactly preserve energy and momentum, Int. J. Numer. Methods Eng. 31 (1991) 19-52.

[20] F. Armero, I. Romero, On the formulation of high-frequency dissipative time-stepping algorithms for nonlinear dynamics. Part I: low-order methods for two model problems and nonlinear elastodynamics, Comput. Methods Appl. Mech. Eng. 190 (2001) 26032649.

[21] Z.Q. Feng, 2D or 3D frictional contact algorithms and applications in a large deformation context, Commun. Numer. Methods Eng. 11 (1995) 409-416.

[22] http://gmfe16.cemif.univ-evry.fr:8080/ feng/FerSystem.html.

[23] Z.Q. Feng, Some test examples of 2D and 3D contact problems involving Coulomb friction and large slip, Math. Comput. Model. 28 (4-8) (1998) 469-477 (Special issue, Recent Advances in Contact Mechanics).

[24] M. Hjiaj, Z.Q. Feng, G. De Saxcé, Z. Mróz, Three-dimensional finite element computations for frictional contact problems with non-associated sliding rule, Int. J. Numer. Methods Eng. 60 (2004) 2045-2076. 